\title{
Surgical treatment of large-cell neuroendocrine cancer of the lung in a patient with situs inversus totalis
}

\author{
Tomasz Karol Prystupa', Dariusz Sagan', Grzegorz Wilczynski', Marek Sawicki' \\ ${ }^{1}$ Department of Thoracic Surgery, Medical University, Lublin, Poland \\ Prystupa T. K, Sagan D, Wilczynski G, Sawicki M. Surgical treatment of large-cell neuroendocrine cancer of the lung in a patient with situs \\ inversus totalis. J Pre-Clin Clin Res. 2018; 12(3): 92-94. doi: 10.26444/jpccr/94397
}

\begin{abstract}
Lung cancer is the most common malignant tumour worldwide. Large-cell neuroendocrine cancer of the lung is its rare form; standard treatment in the early stage involves anatomical resection of the pulmonary parenchyma. In patients with situs inversus, which is a rare congenital condition, the surgical treatment of lung cancer is associated with additional difficulties and an increased risk of cardiovascular complications that should be considered during pre-operative assessment. The report presents the case of a patient with situs inversus totalis who underwent left upper lobectomy due to neuroendocrine lung cancer. The difficulties associated with the procedure are described. Anatomical resection of the pulmonary parenchyma is the optimal surgical treatment for neuroendocrine lung cancer, which can also be applied in patients with situs inversus totalis.
\end{abstract}

\section{Key words}

situs inversus, neuroendocrine cancer, lobectomy

\section{INTRODUCTION}

Situs inversus viscerum is a congenital condition in which the normal arrangement of the viscera is reversed to form a mirror picture of the usual position. In the general population the condition is rare with the incidence of 1:8,500 of live births and affects both genders equally [1]. The majority of patients with situs inversus have cardiovascular and respiratory problems [2].

Patients with situs inversus may develop neoplastic diseases requiring surgical treatment, these include lung cancer, which is the most common malignant tumour worldwide [3] In such cases, additional technical difficulties during surgery should be expected, which are associated with anatomical differences in the structure of internal organs. The risk of peri-operative cardiovascular complications in situs inversus patients is higher $[2,3]$.

\section{CASE REPORT}

A male patient aged 65, a heavy smoker with a history of generalised atherosclerosis, arterial hypertension and ischaemic cerebral stroke, was admitted to the Department of Thoracic Surgery in Lublin due to persistent dry cough, weakness and easy fatigability. Prior to admission, the patient had been treated by his GP for several months without significant improvement. He was referred for a follow-up chest X-ray (Fig. 1) which revealed radiological changes and situs inversus. The patient was unaware of this condition and complicated the treatment of neoplastic proliferation. Bronchoscopy demonstrated a proliferative lesion in the left lung of the right lung anatomy. The material collected during brush biopsy showed the presence of single cells of suspected

Address for correspondence: Tomasz Karol Prystupa, Department of Thoracic Surgery, Medical University, Lublin, Poland

E-mail:Tprym@wp.pl

Received: 3 July 2018; Accepted: 16 August 2018

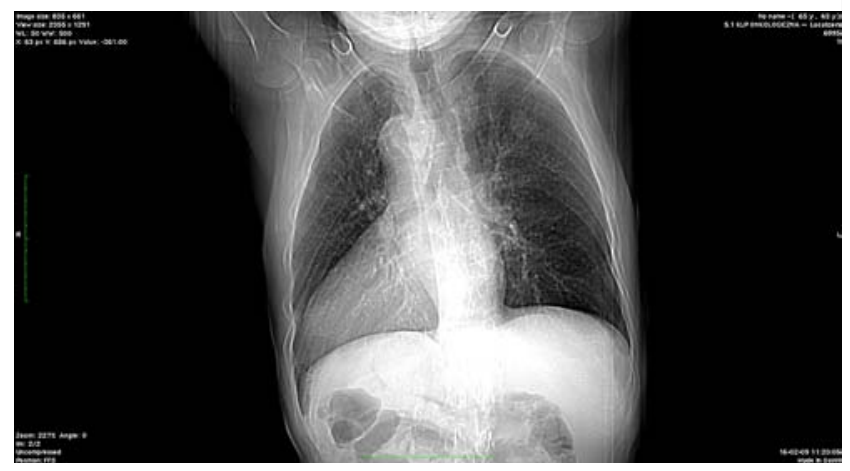

Figure 1. Chest X-ray showing situs inversus totalis and an irregular oval shadow in the upper lobe of the left lung

neoplastic nature. The patient was scheduled for left upper lobectomy. His anatomical condition- CT-confirmed situs inversus of chest organs was taken into consideration (Fig. 2). The patient also had other cardiac problems, including arterial hypertension, which additionally burdened the surgical procedure and could lead to post-operative complications. The surgical team also had to take into consideration his post-stroke condition.

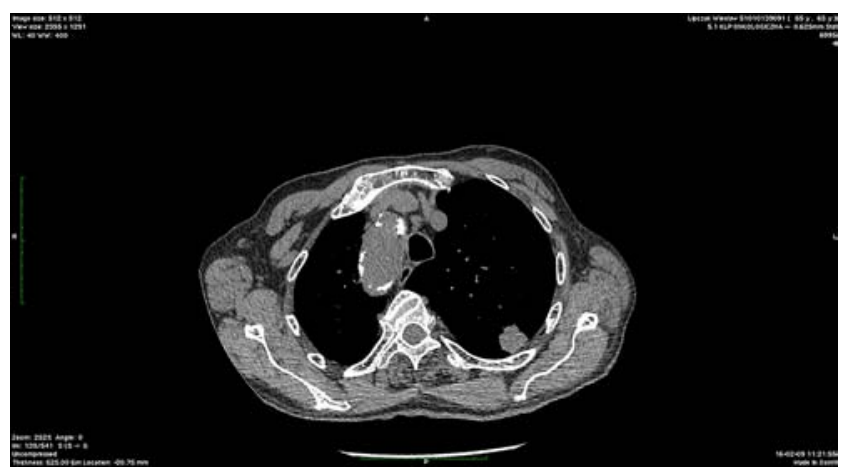

Figure 2. Computed tomography scan disclosing the tumour in the upper lobe of the left lung in a patient with situs inversus totalis 
The surgery was performed at the Department of Thoracic Surgery. The left pleural cavity was opened by lateral thoracotomy; three lobes separated by fissures and a peripheral tumour at S1 and S2 were visualised. Wedge resection was performed and the tumour sent for intraoperative histopathological examination, which revealed poorly-differentiated neuroendocrine cancer. The patient underwent left upper lobectomy. The upper lobe was supplied by two arterial branches - apical and posterior. During preparation of the venous confluence, three separate pulmonary veins were observed, upper-, middle- and lowerlobe, draining to the left atrium through separate orifices. The arteries and veins supplying the upper lobe were closed with vascular staplers and divided. The anterior poorly-developed part of the inter-lobar fissure was separated using a linear stapler. After separation of the upper lobe and removal of lymph nodes, the bronchus leading to the upper lobe was stapled and divided. Areas of air leakage from the pulmonary parenchyma were sealed by sutures and TachoSil. Two drains were inserted into the pleural cavity and the thoracic cavity closed with layered sutures.

The early post-operative course was normal. On postoperative day 4, the patient developed non-ST-segment elevation myocardial infarct (NSTEMI) and was transferred to the Department of Cardiology where he underwent emergency coronarography. The examination demonstrated three-vessel ischaemic heart disease. Moreover, the medial segment of the left anterior descending coronary artery (LAD) was critically constricted and the marginal circumflex branch was occluded below the branching-off of the first branch of the blunt margin of the right coronary artery. Simultaneously, successful angioplasty of the anterior interventricular branch of the left coronary artery was performed with drug-eluting stent (DES) implantation. The course of the procedure and the post-operative period were uneventful. The patient was transferred to the Department of Thoracic Surgery in a stable condition for further management. He was still unconscious and mechanically ventilated and was admitted to the Cardiological Intensive Care Unit; after obtaining family consent, the patient was transported to the District Hospital for further ICU treatment. His further history remains unknown.

\section{DISCUSSION}

Bronchogenic lung cancer is one of the most common malignant tumours both in men and women. The main risk factors of this cancer include tobacco smoking, environmental pollution, chronic lung diseases leading to fibrosis and parenchymal restructuring, as well as genetic predispositions. Lung cancer is characterised by late detection and poor prognosis $[1,2]$. There are two main types of lung cancer: small-cell and non-small-cell lung cancer, which occur in $85 \%$ of all primary lung carcinomas. The earlier it is detected, the better the chances for successful treatment. [4] Neuroendocrine cancers constitute about $20 \%$ of primary malignant tumours located in the lungs [2]. The large-cell type of neuroendocrine non-small-cell cancer belongs to the group of poorly differentiated neuroendocrine tumours requiring aggressive treatment. It is predominantly located peripherally. The standard surgical treatment in the early stage is radical anatomical resection of the pulmonary parenchyma $[3,5]$. Large-cell neuroendocrine cancers are characterised by aggressive course, poor prognosis and high mortality. Their grade of malignancy is determined according to histopathological findings. Situs inversus is a congenital autosomal recessive condition. [6] which manifests in homozygotes. In situs inversus, the thoracic and abdominal organs are arranged in a mirror image, the reverse of the normal positioning $[5,6]$, which leads to frequent complications, mostly cardiac $[6,7]$. This diagnostic challenge requires accurate pre-operative assessment of the location of pulmonary lobes and their relations. In about $25 \%$ of cases, situs inversus coexists with Kartagener's syndrome.

The above abnormalities can be visualised using X-ray and ultrasound. Computed tomography (CT) is preferable to confirm the occurrence of situs inversus. It is important to properly label the sides while planning the thoracic procedures. Available literature contains only a few publications describing the surgical treatment of lung cancer in patients with situs inversus totalis. Most of these reports describe classical lobectomies as if the surgery was performed on the right anatomically normal lung.

In 2009, Bielewicz et al. performed lower bilobectomy of the left lung in a patient with situs inversus and nonsmall-cell cancer [1]. In 2012, Wójcik et al. performed left-sided thoracotomy and laparoscopy in a patient with situs inversus and large-cell cancer of the left lung with metastases to the right adrenal gland [3]. Kodama et al. reported an elderly patient diagnosed with squamous cell cancer of the middle lobe of the left lung who underwent left middle lobectomy with removal of segments S7 and S8, and resection of malignantly infiltrated ribs VI and VII [8]. Apart from the situs inversus, there were no other anatomical abnormalities observed during the procedure. Subotich et al. described the radical treatment of adenocarcinoma of the left lung during which left upper lobectomy was performed. The anatomical relations were found to be normal as on the right side [9].

\section{CONCLUSIONS}

Anatomical resection of the pulmonary parenchyma is the optimal surgical treatment of neuroendocrine lung cancer, which can also be applied in patients with situs inversus totalis.

However, this kind of management is associated with an increased risk of peri-operative complications as such patients frequently have structural anomalies of the lungs, heart and mediastinum. Patients with situs inversus totalis require extremely accurate pre-operative imaging and functional diagnostic procedures to prevent peri-operative complications. Moreover, physical examination and other commonly available imaging examinations are vital for the diagnosis of this rare anatomical condition.

\section{REFERENCES}

1. Bielewicz M, Wojtyś M, Witkowska D, Alchimowicz J, Wójcik J, Grodzki T. Non-small cell lung cancer in patient with visceral total inversion. Pneumon Alergol Pol. 2009; 77(2): 200-204.

2. Langfort R, Rudziński P, Burakowska B. Pulmonary neuroendocrine tumors. The spectrum of histologic subtypes and current concept on diagnosis and treatment. Pneumon Alergol Pol. 2010; 78(1): 33-46. 
3. Wójcik J, Kubisa, Pieróg J, Bielewicz M, Bielewicz M, Wójcik N, Kaseja $\mathrm{K}$, Grodzki T. Lung cancer in situs inversus totalis (SIT) - a case report. Kardiochir Torakochir Pol. 2012; 9(2): 219-221.

4.Langfort R. Rola badań patomorfologicznych w wyborze metody leczenia raka płuca. Onkol Dypl J. 2016; 13(1): 42-47.

5. Mitura K, Romańczuk M, Blicharz P, Boruciński M. Laparoscopic cholecystectomy In patient with situs viscerum inversus. Wideochir Tech Maloinwaz. 2006; 1(1): 40-42.

6. Yoshida M, Hino H, Machida H, Hatakeyama N, Okano Y, Iwahara Y, Shinohara T, Oogushi F. Video-assisted thoracic surgery lobectomy for lung cancer in a patient with complete situs inversus. Gen Thorac Cardiovasc Surg. 2013; 61: 155-159.

7. Rydel M, Czyżewski D, Stęplewski K, Zygo A, Adamek M. Synchronous two distinct neuroendocrine lung cancer lesions. Pneumon Alergol Pol. 2015; 83(3): 212-215.

8. Kodama K, Doi O, Tatsuta M. Situs inversus totalis and lung cancer. Chest. 1990; 97: 1274-1275.

9. Subotich D, Mandarich D, Katchar V, Bulajich B, Drnarski B. Lung resection for primary bronchial carcinoma in a patient with complete situs inversus. Clin Anat. 2006; 19: 358-362. 